\title{
Inversión extranjera directa: su incidencia en la tasa de empleo del Ecuador
}

\section{Direct foreign investment: its incidence in the employment rate of Ecuador}

\author{
Jéssica Alexandra Espin* \\ jsy_24@hotmail.com \\ Ana Consuelo Córdova** \\ anaccordova@uta.edu.ec \\ Gonzalo Efraín López*** \\ gozhalo@yahoo.com
}

\begin{abstract}
Resumen
El objeto de este estudio consistio en establecer la incidencia que tuvo la Inversión Extranjera Directa, IED, en la Tasa de Empleo del Ecuador en el periodo 2007-2014 y se realizó en base a los indicadores emitidos por el Banco Central del Ecuador y el INEC. Esta investigación fue de tipo correlacional, se realizó a través de una regresión lineal simple, dónde se evidenció que la IED no incide en la Tasa de Empleo por actividad económica, en lo posterior se construyó un modelo econométrico con las variables: PIB, Salario Real e IED. Los resultados demostraron que sólo el PIB y Salario Real son estadísticamente significativos con respecto a la Tasa de Empleo, determinando que la IED como única variable regresora no fue un factor determinante en el Empleo durante el periodo establecido.
\end{abstract}

\section{Palabras clave}

Economía, inversión extranjera directa, tasa de empleo

* Ecuatoriana. Economista. Banco de Desarrollo del Ecuador B.P.- Ambato. Secretaria Ejecutiva.

** Ecuatoriana. Magíster en Gerencia Financiera Empresarial. Universidad Técnica de Ambato. Directora Administrativa Financiera.

*** Ecuatoriano. Economista. Artesanía y Procesadora de Lácteos ARPAL. Píllaro. Jefe de Comercialización y Ventas. 


\begin{abstract}
The purpose of this study consisted on establishing the incidence of Foreign Direct Investment (FDI) in the Employment Rate of Ecuador in the period 2007-2014 and was based on the indicators issued by the Central Bank of Ecuador and the INEC. This research was of a correlational type, it was done through a simple linear regression, where it was evidenced that the FDI does not affect the Employment Rate by economic activity, in the later it was constructed an econometric model with the variables: GDP, Real Salary And FDI. The results showed that only GDP and Real Salary are statistically significant with respect to the Employment Rate, determining that FDI as the only regressive variable was not a determinant factor in Employment during the established period.
\end{abstract}

\title{
Keywords
}

Direct foreign investment, economy, employment rate.

Forma sugerida de citar: Espín, Jéssica Alexandra, Córdova, Ana Consuelo, \& López, Gonzalo Efraín (2016). Inversión extranjera directa: su incidencia en la tasa de empleo del Ecuador. Retos, 12(2), pp.215-228.

\section{Introducción}

En un mundo globalizado, la inversión entre países varía acorde a las necesidades de las potencias mundiales. Los países receptores de dicha inversión promueven políticas atrayentes para captar ingresos extranjeros mediante diversos acuerdos como: fortalecimientos institucionales, aranceles, acceso a mercados locales con mayor facilidad, mejoramiento de relaciones externas y otras. De este modo, todos los países mantienen al menos un tipo de relación comercial con otro país.

La inversión se destina a una diversa gama de sectores y actividades económicas, sin embargo, el im- pacto que genere en una no se verá reflejado en la misma magnitud en otro sector económico.

La IED, brinda apertura a tratados internacionales que buscan la liberalización comercial y el consenso de políticas que beneficien a los países miembros de tales alianzas. A nivel mundial, se determinan numéricamente altos valores alcanzados en IED, pero no se determinan sectores, países o regiones beneficiados en igualdad de condiciones, esto depende del destino de la IED, la demanda internacional de los productos y su máxima explotación.

En el año 2013 las expectativas de IED mundial fueron superadas a 
las planteadas en un inicio, principalmente por los países en vías de desarrollo, representando más del $50 \%$ del total de las entradas de IED (CEPAL, 2013, p. 20)

A lo largo de la historia de la IED se ha evidenciado que favorece a países en crisis económicas, ya que inyecta capital para impulsar la producción, aumento del empleo y por ende el incremento del PIB, que es considerado un indicador revelador de una economía.

Al considerar la época de los años 90, surgieron infinidad de acuerdos internacionales y Estados Unidos se convirtió en el gran inversor del mundo entero. Los países de América Latina eran los elegidos por la cantidad de recursos naturales que poseían.

La consolidación de los países en macro-regiones se fundamenta en acuerdos internacionales comerciales, donde los beneficios incrementan debido al incentivo entre los países miembros, enfocados en políticas económicas que merman los riesgos inherentes al invertir en un país extranjero.

En América Latina el flujo de IED se centra en servicios y manufacturas a excepción de países con abundancia de recursos naturales, donde los inversionistas se enfocan en el sector minero e hidrocarburos, siendo el caso de Brasil, Colombia, Ecuador y Venezuela.
En el país, la IED ha presentado diversas fluctuaciones, sin embargo, en los últimos años, la misma no ha mostrado un ingreso considerable según lo indican los datos del BCE y que se analizan posteriormente.

El Econ. Rafael Correa, Presidente de la República, estableció que la IED será recibida en el Ecuador siempre y cuando no sea un "Capital Golondrina", sino que se quede en el país y su estancia genere beneficios, por lo tanto, al fundamentar su posición en tal situación se puede asegurar la permanencia de dicho capital al menos por un tiempo necesario para reflejar aspectos económicos y sociales lo suficientemente sustanciales.

A su vez, un determinado número de empresarios exigen incentivos para atraer capitales extranjeros y contrarrestar el desempleo así como el crecimiento de la producción en el país. Por lo tanto, existen posturas antagónicas con respecto a la IED.

Teóricamente la IED es un ente dinamizador de la economía que se convierte en fuente de financiamiento externo: capacita la nueva fuerza laboral, desarrolla procesos productivos, incrementa la tecnología y mejora la relación comercial, generando países competitivos; generando equilibrio en la balanza de pagos de los países y beneficiando a sus economías. 
Sin embargo en el Ecuador se cuestiona lo siguiente: ¿Por qué no se han creado más incentivos para atraer la IED? ¿Qué sucede con las políticas de comercio exterior? ¿Las políticas aplicadas no son suficientes para los inversionistas internacionales? ¿Se está negando una fuente de ingresos al país? $\mathrm{O}$ simplemente, ¿La teoría no va de la mano de la realidad y su impacto no resulta influyente en el país?

Sin la información suficiente, apropiada y correctamente analizada se pueden generar criterios que no estarían bien sustentados y que no podrán contribuir positivamente a tomar decisiones adecuadas en el Ecuador.

La IED en el Ecuador durante los últimos años y en relación a la magnitud de su economía es considerada una de las más bajas de la región, sin embargo, es válida la comparación con el resto de las economías sudamericanas centrándose en el crecimiento del PIB, mismo que no presencia grandes brechas a pesar de las cuantiosas inversiones recibidas por los países vecinos.

Cabe mencionar que existen grandes empresas multinacionales en el Ecuador pese a las duras críticas recibidas por los siguientes motivos: inadecuada infraestructura, elevados costos de los servicios públicos, normativa legal rígida en el ámbito laboral y bajo desarrollo de políticas aperturistas.

Al analizar la IED en el Ecuador durante el período 2007-2014, el año 2013 presentó un alto ingreso, motivado por el incremento de las contribuciones de capital, y en menor porcentaje la extracción petrolera, seguida por manufactura y el comercio interno. Cabe mencionar que los inversionistas del país en el mencionado año fueron: España, Italia, y China.

Es necesario identificar la cantidad de IED que el país receptó durante el periodo establecido y el impacto que generó en las tasas de empleo de los sectores económicos a los que fue destinada; finalmente se propone un modelo econométrico en el que se consideran las variables regresoras: PIB, Salario Real y por efectos de la investigación la IED.

Existen estudios previos que difieren unos de otros dependiendo del país o de la región donde se aplique:

Barreno (2015), en un simulador de impactos "Construcción Matriz Insumo - Producto 2010: Industria - Industria" hace referencia a la importancia de crear políticas que se direccionen al incremento de empleo, además establece que el sector de minas y canteras ha sido el que mayor atracción de inversión ha provocado, y donde paradójicamente la cantidad de plazas de 
trabajo son mínimas, debido a los elevados sueldos que se producen.

En el estudio realizado por (Barraza, 2013, p. 80), "Comportamiento de la IED en Colombia entre el periodo 2000 a 2012 y los efectos esperados a raíz del Tratado De Libre Comercio entre Colombia - Estados Unidos y Colombia - Canada" establece que la Inversión Extranjera Directa fue dirigida al sector mineroenergético y menciona que aunque no sea fuente generadora de empleo, puede generarlo de manera indirecta a través de las actividades que se relacionan con esta actividad, tal es el caso de los gastos de los hogares que aumentaron sus ingresos gracias al incremento de las remuneraciones en la minería.

(Brossard, 2015) establece aspectos positivos y negativos referente al ingreso de IED, es así que, pone en tela de duda la creación de empleos al plantear el ingreso de tecnologías por parte de empresas multinacionales que reemplazarán la mano de obra y dónde no se compensará la pérdida de trabajos debido a la competitividad con que se presentan estas empresas (p. 20)

(Pérez \& Cesín, 2016), en sus conclusiones finales plantean que el sector de industrias manufactureras a través de su mano de obra puede presenciar competitividad laboral tanto para el mercado interno como externo. (p. 170).
Martínez (s.f) analiza el impacto del Tratado de Libre Comercio junto con la IED en el Empleo del Ecuador y manifiesta que un efecto positivo sobre la tasa de empleo está mal infundada ya que abarca nuevos problemas en otros sectores de la economía, especialmente en el sector rural, debido a la migración interna que se presentaría en el país por la presencia de empresas multinacionales que ofrecen productos más económicos y generan mayores tasas de desempleo o en su defecto, precarización laboral; incrementando la informalidad en el mercado ocupacional.

En definitiva, los resultados en cada caso dependen: del tipo de inversión, país de destino de la inversión, apertura comercial y características de las empresas.

\section{Empleo}

Las personas con empleo son aquellas que se encuentran en edad de trabajar, lo realizan, y a cambio perciben una retribución económica.

(INEC, 2014), el empleo presenta diversos criterios para determinar su condición de actividad:

- Desear trabajar horas adicionales.

- Estar disponible para trabajar horas adicionales.

- Ingresos laborales.

- Jornada laboral. 


\section{Empleo adecuado}

Catalogados en este grupo quienes "No tienen insuficiencia en el ingreso y jornada laboral" (INEC, 2014).

\section{Inversión Extranjera Directa}

Es un indicador propio de una Economía abierta, propio de la Globalización actual.

Jiménez (2013):

Desde hace varios años se ha considerado a la inversión extranjera directa como la mayor fuente de entrada de fondos a los países en desarrollo por un pequeño grupo de países emergentes como China, Brasil y otros, que representan el grueso de los recientes incrementos del IED.

Pero las entradas de IED están acompañadas de grandes salidas bajo la forma de repatriación de ganancias. Muchas veces en un periodo de tiempo determinado, las entradas de fondos a través de nuevas inversiones extranjeras directas, en países en desarrollo, fueron superadas por las salidas de fondos como remesas de ganancias sobre el IED existente.

Por otro lado, se establecen modalidades de Inversión según el Banco Central del Ecuador (BCE):

A través de inversión en el capital social de la empresa mediante constitución o adquisición de empresas, denominadas empresas de IED.
También, a través de la incorporación las utilidades no distribuidas al patrimonio de estas empresas.

Finalmente, mediante deuda entre empresas afiliadas o relacionadas; esta modalidad de inversión se denomina "Otro Capital de IED".

Tomando en cuenta las modalidades de Inversión se crean escenarios para establecer a través de cuál de ellos se generaría mayor incidencia en la Tasa de empleo.

La búsqueda de beneficios a través de la IED determina la competencia entre los países, generando estrategias de liberalismo económico para convertirse en un país atractivo.

\section{Producto Interno Bruto}

Que se ha utilizado en el modelo econométrico, según el BCE:

Es el valor de los bienes y servicios de uso final generados por los agentes económicos durante un período. Su cálculo -en términos globales y por ramas de actividad- se deriva de la construcción de la Matriz Insumo-Producto, que describe los flujos de bienes y servicios en el aparato productivo, desde la óptica de los productores y de los utilizadores finales.

\section{Salario real}

Es la remuneración del trabajo medido en términos del poder adquisitivo, es decir, éste se ve afectado por la inflación. 


\section{Metodología}

Esta investigación es de tipo correlacional, se aplicó el método de Mínimos Cuadrados Ordinarios para determinar la relación existente entre las variables. Es importante mencionar que se usaron logaritmos con el propósito de lograr la estacionalidad de los datos y obtener resultados libres de problemas econométricos.

Una vez realizado el contraste de la Tasa de Empleo y la IED por Actividad Económica se planteó un modelo econométrico, en el que se incluye a IED por efectos de estudio, además del PIB y Salarios reales presentados en el periodo de análisis, obteniendo la siguiente ecuación de regresión múltiple:

Ts. empleo $=\beta 0+\beta 1$. PIB $+\beta 2$. IED $+\beta 3$. Salarioreal $+u i$

Posteriormente al análisis de Mínimos Cuadrados Ordinarios se realizó el Modelo de Cointegración de Engle y Granger, el cual según (Montero, 2013), enuncia que
"Cointegración significa que existe una relación, a largo plazo, entre las variables" (p.7).

Todo lo mencionado con ayuda del software econométrico Gretl.

\section{Base de Datos utilizada}

Debido al análisis macroeconómico que se realizó, se hizo uso de la información emitida por el INEC con la aplicación de la metodología ENEMDU - Encuesta Nacional de Empleo, Desempleo y Subempleo a nivel Nacional, en este caso se uso la Tasa de Empleo para su uso en la caracterización por sectores de la economía y la Tasa de Empleo Adecuado y mediante la plataforma electrónica en el Sistema de Información Macroeconómica BI del Banco Central.

\section{Resultados}

$\mathrm{Al}$ analizar el comportamiento de la IED se obtuvieron los siguientes resultados:

\section{Gráfico 1. IED recibida (2007-2014)}

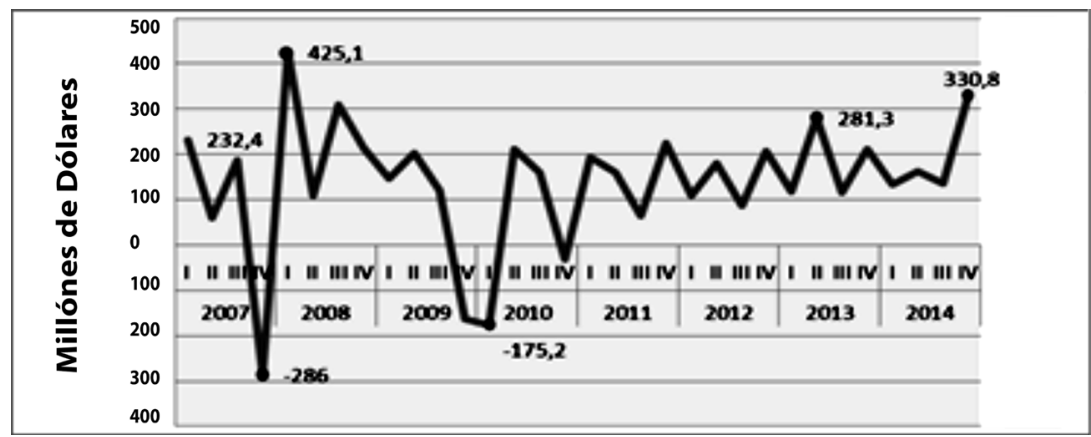

Fuente: BCE

Elaboración propia 
Se observa que en el último trimestre del año 2007, la inversión decae abruptamente, y el país deja de percibir 286 millones de dólares, seguido por un repunte en el primer trimestre del año 2008, cuando 425.1 millones de dólares fueron recibidos como IED.
Durante los años 2011-2014 se observa un crecimiento constante hasta culminar el año 2014 con un ingreso neto de 330.8 millones de dólares en inversión. El gráfico 2 presenta la IED por modalidad de inversión:

\section{Gráfico 2. IED por modalidad de inversión (2007-2014)}

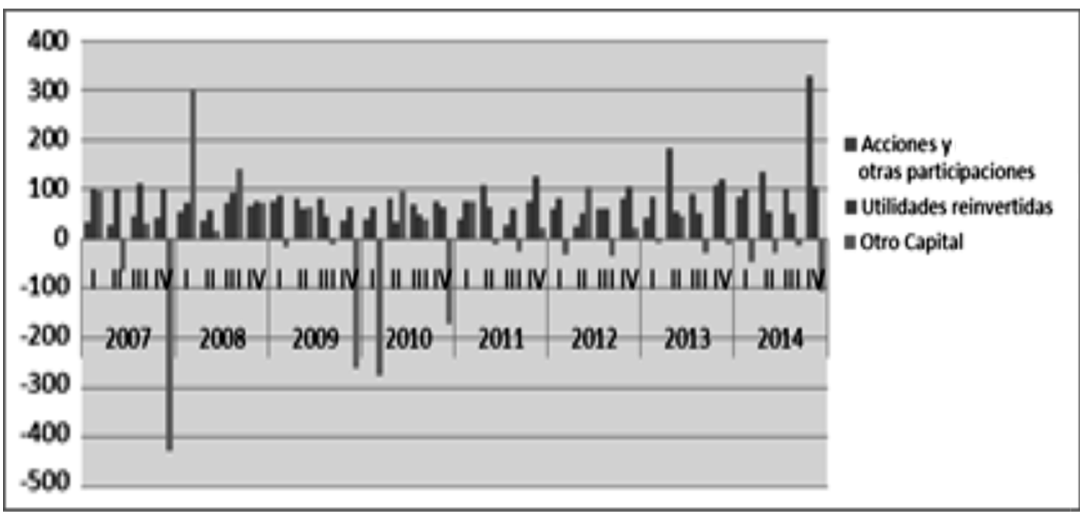

Fuente: BCE

Elaboración propia

Los valores denominados acciones y otras participaciones presentan tendencia constantes, pero existen dos puntos importantes durante el periodo: al inicio del segundo trimestre del año 2013 ingresan 182 millones de dólares, es decir: este tipo de capital incrementó fuertemente durante ese trimestre bajo esta modalidad.

Las utilidades reinvertidas denotan estabilidad en sus ingresos anuales en el año 2011, el Ecuador percibió 126 millones de dólares; significando confianza por parte de los países inversionistas al decidir seguir trabajando en el país.

Bajo la modalidad Otro Capital, existen valores negativos, considerados como "des-inversión" a excepción del 2008, cuando se percibió 299.7 millones de dólares.

$\mathrm{Al}$ analizar la IED y su impacto en las actividades económicas se encontraron dos casos puntuales en dos sectores económicos:

El gráfico 3 muestra la variación estimada del Empleo con respecto a la IED en la Agricultura. 
Gráfico 3. Variación estimada del Empleo con respecto a la IED - Agricultura

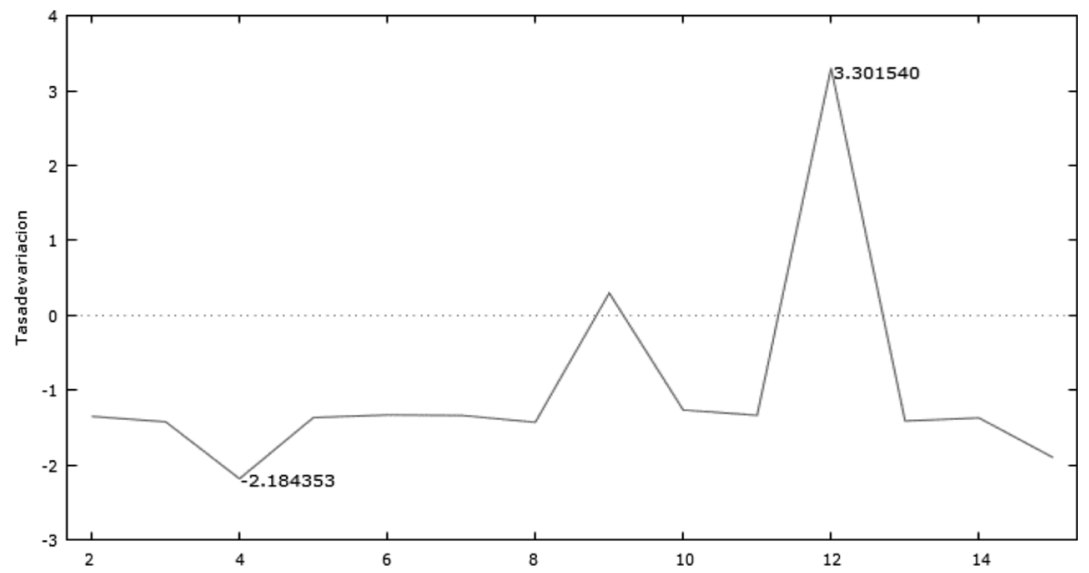

Fuente: Gretl

Elaboración propia

La relación existente de esta actividad económica con la IED fue del $11 \%$, y generó una variación máxima de 3.3 puntos porcentuales en el año 2013 y una variación negativa de -2.18 en el año 2009.

El gráfico 4 muestra la variación estimada del Empleo con respecto a la IED en el Comercio.

\section{Gráfico 4. Variación estimada del Empleo con respecto a la} IED - Comercio

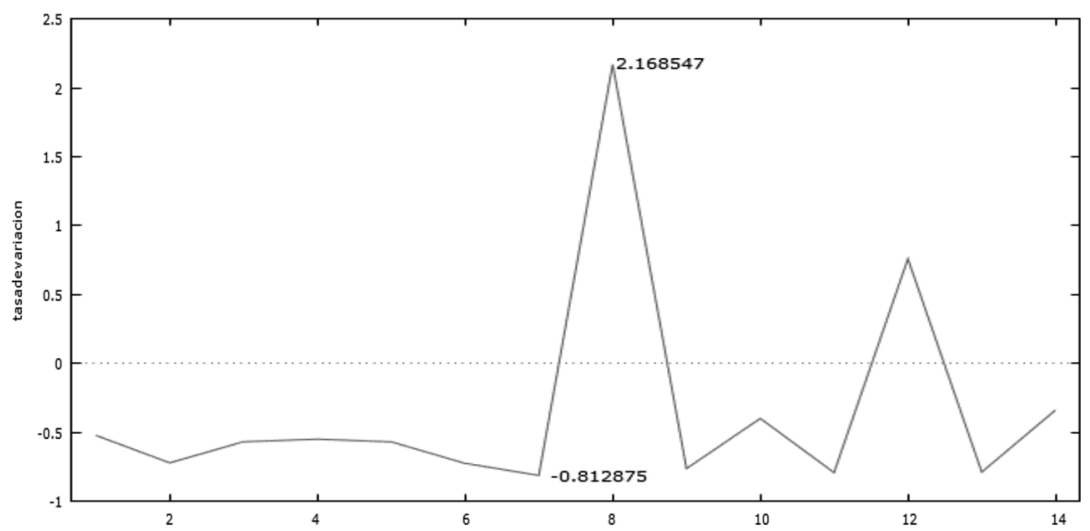

Fuente: Gretl

Elaboración propia 
La relación que se presenta en esta actividad económica con la IED fue del 7\%, y generó una variación máxima de 2.16 puntos porcentuales en el 2011 y una variación negativa casi nula de -0.81 puntos porcentuales en el segundo semestre del 2010.
Con respecto al resto de actividades económicas se verificó que la relación existente de cada una de ellas con la IED fue menor al $2 \%$.

La tabla 1 muestra la matriz de correlación entre las variables.

\section{Tabla 1. Matriz de correlación}

\begin{tabular}{|l|l|l|l|}
\hline \multicolumn{4}{|c|}{$\begin{array}{c}\text { Correlation Coefficients, using the observations 2:1 - 8:2 } \\
\text { (missing values were skipped) }\end{array}$} \\
\hline 1_EmpleoAdecua- & 1_PIB 1_IEDMillonesd- & \multicolumn{1}{c|}{ 1_Salario } \\
\hline 1.0000 & 0.8238 & 0.3427 & 0.5193 1_EmpleoAdecua- \\
\hline & 1.0000 & 0.4772 & 0.4166 1_PIB \\
\hline & & 1.0000 & 0.5498 1_IEDMillonesd- \\
\hline & & & 1.0000 1_Salario \\
\hline
\end{tabular}

Fuente: Software Gretl

Elaboración propia

Se presentaron los siguientes resultados:
- Correlación poco significativa entre el Empleo y los Salarios reales.

- Correlación altamente signifiLa tabla 2 muestra los resultacativa entre el Empleo y el PIB. dos obtenidos de la regresión Lineal

- Correlación no significativa múltiple a través del Software ecoentre el Empleo y la IED. nométrico Gretl:

Tabla 2. Modelo Mínimos Cuadrados Ordinarios Log-Log Modelo 1: MCO, usando las observaciones 1:2 - 8:2 ( $T=12)$ Se han quitado las observaciones ausentes 0 incompletas: 3 Variable dependiente: $1 \_Y$

\begin{tabular}{|l|l|l|l|lc|}
\hline & \multicolumn{1}{|c|}{ Coeficiente } & $\begin{array}{r}\text { Desv. } \\
\text { Típica }\end{array}$ & \multicolumn{1}{|c|}{ Estadístico t } & \multicolumn{2}{|c|}{ Valor $p$} \\
\hline const & $-13,2016$ & 5,23617 & $-2,521$ & $0,0357 \quad{ }^{* *}$ \\
\hline 1_x1 & 6,77788 & 1,92228 & 3,526 & 0,0078 & *** \\
\hline $1 \_x 2$ & $-1,06494$ & 0,736011 & $-1,447$ & 0,1859 & \\
\hline 1_x3 & 0,492429 & 0,174718 & 2,818 & $0,0225 \quad *$ \\
\hline
\end{tabular}




\begin{tabular}{|lcll|}
\hline Media de la vble. dep. & 3,802066 & D.T. de la vble. dep. & 0,062430 \\
Suma de cuad. resiuos & 0,006802 & D.T. de la regresión & 0,029160 \\
R-cuadrado & 0,841337 & R-cuadrado corregido & 0,781838 \\
F $(3,8)$ & 14,14043 & Valor p (de F) & 0,001457 \\
Log-verosimilitud & 27,82514 & Criterio de Akaike & $-47,65029$ \\
Criterio de Schwarz & $-45,71066$ & Crit. de Hannan-Quinn & $-48,36840$ \\
\hline
\end{tabular}

Fuente: Software Gretl

Elaboración propia

De acuerdo al modelo de regresión lineal expuesto anteriormente se observa que mediante el incremento de un punto porcentual en la variable PIB (X1), la tasa de empleo Adecuado aumentará en 6.7 puntos porcentuales. En la variable IED (X2) por cada punto porcentual que se incremente, la tasa de variación del Empleo disminuirá en 1.06 puntos porcentuales. Finalmente, por cada punto porcentual que se incremente en la tasa de va- riación de los Salarios Reales la tasa de empleo incrementará en 0.49 puntos porcentuales.

En este modelo de regresión se determina que los estimadores de las variables PIB (X1) y Salarios Reales (X3) son estadísticamente significativos, mientras que el estimador de la variable IED (X2) no tiene significación estadística.

Posteriormente se analizó si existe presencia de Cointegración a largo plazo.

Tabla 3. Modelo Mínimos Cuadrados Ordinarios Log-Log

\begin{tabular}{|c|c|c|c|c|c|}
\hline \multicolumn{6}{|c|}{ Etapa 5: regresión cointegrante } \\
\hline \multicolumn{6}{|c|}{ Regresión cointegrante - } \\
\hline \multicolumn{6}{|c|}{ MCO, usando las observaciones 1:2 - 8:2 ( $T=12)$} \\
\hline \multicolumn{6}{|c|}{ Se han quitado las observaciones ausentes o incompletas: 3} \\
\hline \multicolumn{6}{|c|}{ Variable dependiente: I_Y } \\
\hline & Coeficiente & Desv. Típica & Estadístico t & \multicolumn{2}{|c|}{ Valor $p$} \\
\hline const & $-13,2016$ & 5,23617 & $-2,521$ & 0,0357 & ** \\
\hline $1 \_x 1$ & 6,77788 & 1,92228 & 3,526 & 0,0078 & *** \\
\hline 1_x2 & $-1,06494$ & 0,736011 & $-1,447$ & 0,1859 & \\
\hline $1 \_\times 3$ & 0,492429 & 0,174718 & 2,818 & 0,0225 & ** \\
\hline
\end{tabular}

Fuente: Software Gretl

Elaboración propia 
Se observa que 1_x1 (PIB) y 1_x3 (Salario real), presentaron Cointegración a largo plazo, mientras que 1_x2 (IED) no presentó Cointegración a largo plazo en el periodo establecido.

\section{Discusión}

(Bonilla, 2010) en la investigación realizada en Colombia sobre la verdadera participación del sector externo en la Ocupación durante los años 1985-2006, la IED no fue significativa, este resultado lo obtuvieron a través de una regresión múltiple con $\mathrm{MCO}$. A partir de la apertura al sector externo y principalmente al ingreso de IED no se observó impactos positivos en el crecimiento económico sino desequilibrios en el mercado de trabajo y la tasa de desempleo incrementó.

CEPAL (2013) teóricamente la IED se puede transformar en motor de la economía de los países que la reciben, sin embargo hace énfasis en la dependencia del sector-destino de esta inversión; menciona la creación de un puesto de trabajo por cada millón de dólares en Minas y Petróleos, donde la cantidad de recursos naturales a ser explotados en América Latina son incalculables. Además establece que durante el periodo 2003-2013, la IED recibida ha originado como creación neta de empleos en América del Sur apenas un $5 \%$.
Romero (2012) en referencia al estudio realizado por Arellano y Bond cuyo resultado sobre el impacto de la IED en el empleo de trabajadores calificados y no calificados obtenido a través del método generalizado de momentos (MGM), establece que éste es significativamente positivo pero cuantitativamente modesto en la generación de empleo en México, determinando que los efectos que trae la IED en el empleo depende de muchos factores.

En esta investigación se observan resultados similares a los obtenidos en Colombia, debido a que la IED no presenta un grado de correlación significativa e incide con la Tasa de Empleo, situación contraria se evidencia con el PIB, que presenta correlación significativa y e incide positivamente en el aumento del Empleo.

Un caso contrario sucede con el segundo estudio presentado en la discusión de este proyecto debido a que la correlación es positiva, pero no representa una incidencia considerable en la tasa de empleo en la maquila en México.

\section{Conclusiones}

La IED en el Ecuador, no superó los 425 millones de dólares durante el periodo de análisis, que en comparación con los países vecinos es relativamente baja, sin embargo, la modalidad que mayor ingresos re- 
presentó fue la de utilidades reinvertidas, es decir, los inversionistas al cúlmino de su contrato, decidieron reinvertir nuevamente en el país.

En cuanto a los efectos de la IED en el empleo establecido por ramas de actividad económica, la mayor influencia que posee la IED es en la Agricultura, estimando una tendencia negativa y expresando una relación inversamente proporcional, siendo que la influencia de ésta, aunque mínima, incide en esta rama con respecto a las demás; esto se debe a que la IED en lugar de crear puestos de trabajo reemplaza la mano de obra por tecnología.

El análisis realizado en los sectores de Minas y Petróleos, Manufactura, Suministro de Electricidad, Gas y Agua, Construcción, Transporte y Comunicación reflejaron una mínima incidencia de la IED en el Empleo (motivo por el que no han sido plasmados en la presente investigación), esto se debe a que la IED no contribuyó de forma significativa en estos sectores, siendo que la mayor parte de la inversión que estos reciben provienen de la iniciativa micro-empresarial y estatal.

Por su parte, en el comercio, el empleo se explica en un $7 \%$ por la IED, demostrando que la inversión más influyente en el sector podría ser la inversión nacional.

Posterior a la experimentación empírica del modelo econométrico del Empleo Adecuado bajo MCO no se mostraron relaciones estadísticamente significativas; y un modelo de Cointegración tampoco logro explicar relación alguna a largo plazo entre la IED y el Empleo.

A manera de conclusión global se establece que la IED como única variable independiente no explicó a la Tasa de Empleo en el período establecido; los resultados pueden diferir tomando en cuenta el período de análisis, la situación del país, región o lugar, el nivel de industrialización y diversos factores que incidan determinantemente en los resultados.

\section{Bibliografía}

Barraza, Z. (22 de Noviembre de 2013). Comportamiento de la IED en Colombia entre el Periodo 2000 a 2012 y los efectos esperados a raíz del Tratado de Libre Comercio entre Colombia-Estados Unidos y Colombia-Canada. Obtenido de Repositorio digital de La Universidad Militar "Nueva Granada": http://repository.unimilitar. edu.co/bitstream/10654/11401/2/ PROYECTO\%20ZULLY\%20 cod\%203401238.pdf

Barreno, A. (2015). Tesis de pregrado. Recuperado en Septiembre de 2015, de Pontificia Universidad Católica del Ecuador - Repositorio Digital: http://repositorio. puce.edu.ec/handle/22000/8394

Bonilla, E. (2010). El sector externo y su incidencia en la ocupación en Colombia 1985-2006. , 83-98. 
BCE (s.f.). Banco Central del Ecuador. Obtenido de www.bce.fin.ec (s.f.). Obtenido de

http://contenido.bce.fin.ec/documentos/Estadisticas/SectorExterno/ BalanzaPagos/MetodoCambioComBP.pdf

Brossard, D. (Julio de 2015). La Inversión Extranjera Directa. Su necesidad para el desarrollo en economías seleccionadas. Obtenido de Repositorio Institucional Scriptorium. Universidad de La Habana: http://dspace.uh.cu:8080/xmlui/ handle/123456789/2715

Comisión Económica para América Latina y el Caribe (CEPAL) (2013). La Inversión Extranjera Directa en América Latina y el Caribe (LC/G.2613-P). Santiago de Chile, 2014.

INEC (Octubre de 2014). Nuevo marco conceptual para la clasificación del empleo en Ecuador. Obtenido de www.ecuadorencifras.gob.ec

Jiménez, A. (2013). Mister ¿Por qué nosotros? Estados Unidos.

Martínez, L. (s.f.). . Obtenido de http:// www.flacso.org.ec/docs/TLCyEmpleo.pdf

Montero, R. (2013). Variables no estacionarias y cointegración. Documentos de Trabajo en Economía Aplicada. España: Universidad de Granada.

Pérez, S., \& Cesín, A. (2016). Obtenido de http://www.ecorfan.org/handbooks/Handbook_Matematicas_Aplicadas_a_la_Economia_ T1V1/Particiones/13.pdf

Romero, J. (2012). Inversión extranjera directa y crecimiento económico en México, 1940-2011. , 109. 147.

Recepción: 07-19-2016-Aceptación: 12-06- 2016. 